\title{
Acinic cell carcinoma of the nasal vestibule. Report of a unique case*
}

\author{
Argyris Manganaris ${ }^{1}$, Timothy John Bates ${ }^{2}$, David Roberts ${ }^{1}$, Ricard Simo \\ Department of Otorhinolaryngology - Head and Neck Surgery, Guy's and St Thomas' Hospital NHS \\ Foundation Trust, Guy's Hospital, London, United Kingdom \\ 2 Department of Oral Pathology, Guy's and St Thomas' NHS Foundation Trust, Guy's Hospital, London, \\ United Kingdom
}

\section{SUMMARY}

\begin{abstract}
Glandular tumours constitute a very small percentage of all neoplastic lesions in the nasal cavity. Only a very limited number of cases of acinic cell carcinoma (ACC) involving the nose have been sporadically reported throughout the medical literature. The clinical significance of this case report is due to its unique site. To our knowledge this report represents the only documentation of acinic cell carcinoma arising in the nasal vestibule. Unusual presentations of uncommon malignancies can pose considerable diagnostic dilemmas to both the surgeon and histopathologist. Awareness of these rarities is important to ensure the best patient care.
\end{abstract}

Key words: minor salivary gland, acinic cell carcinoma, nasal vestibule, conchal graft, nasal reconstruction

\section{INTRODUCTION}

The majority of minor salivary glands are found in the oral cavity. Similar salivary tissue is found in the nose, paranasal sinuses and nasopharynx. The acinar structure of these glands is very similar to the major salivary glands but the ductal system is relatively simpler. The incidence of minor salivary gland tumours (MSGT) varies in relation to each anatomical site of the airway. In a 35-year review of 2,807 patients from 1986 Spiro et al. reported that the most common site was the oral cavity, accounting for $70.1 \%$, followed by the nose and paranasal sinuses, accounting for $26.8 \%{ }^{(1)}$.

MSGT account for $4 \%$ to $8 \%$ of all sinonasal malignancies ${ }^{(1,2)}$. Adenoid cystic carcinoma is the most common histological type, followed in decreasing incidence by adenocarcinoma, pleomorphic adenoma, mucoepidermoid carcinoma, and undifferentiated carcinoma ${ }^{(1,2)}$. In the sinonasal tract MSGT are often misdiagnosed as polyps or infectious ulcers. However, they tend to behave aggressively, regardless of histological type. Consequently, MSGT in this area tend to have a poor prognosis, which is directly related to the delayed diagnosis.

ACC arising in the nose is exceptionally rare, and is therefore clinically unsuspected in the initial evaluation of a patient presenting with a polypoidal mass or nasal ulcer. The clinical data concerning ACC of the sinonasal tract is very limited compared to other more common histological types. Therefore, the clinical course, optimal treatment and prognosis have not been extensively studied. The aim of this report, besides presenting a unique case, is to summarize all the key points, following a comprehensive literature review.

\section{CLINICAL REPORT}

A 51-year-old female presented with a six-month history of localized pain in the left nasal cavity, without a previous history of lesion at the same site. No history of nasal obstruction, rhinorrhoea, epistaxis, or anosmia was given. The patient's medical and family history were non-contributory.

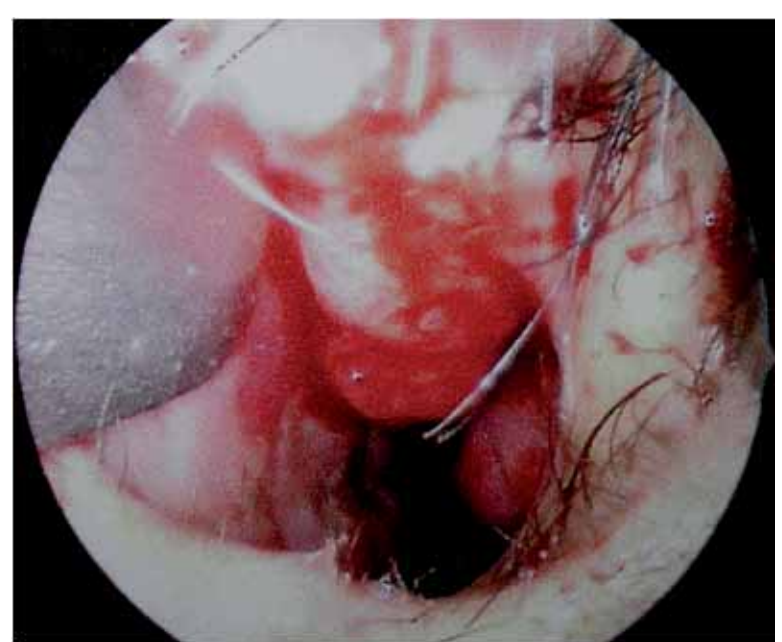

Figure 1. Pre-operative tumour appearance. Anterior Rhinoscopy shows the ulceration at the left nasal vestibule. 


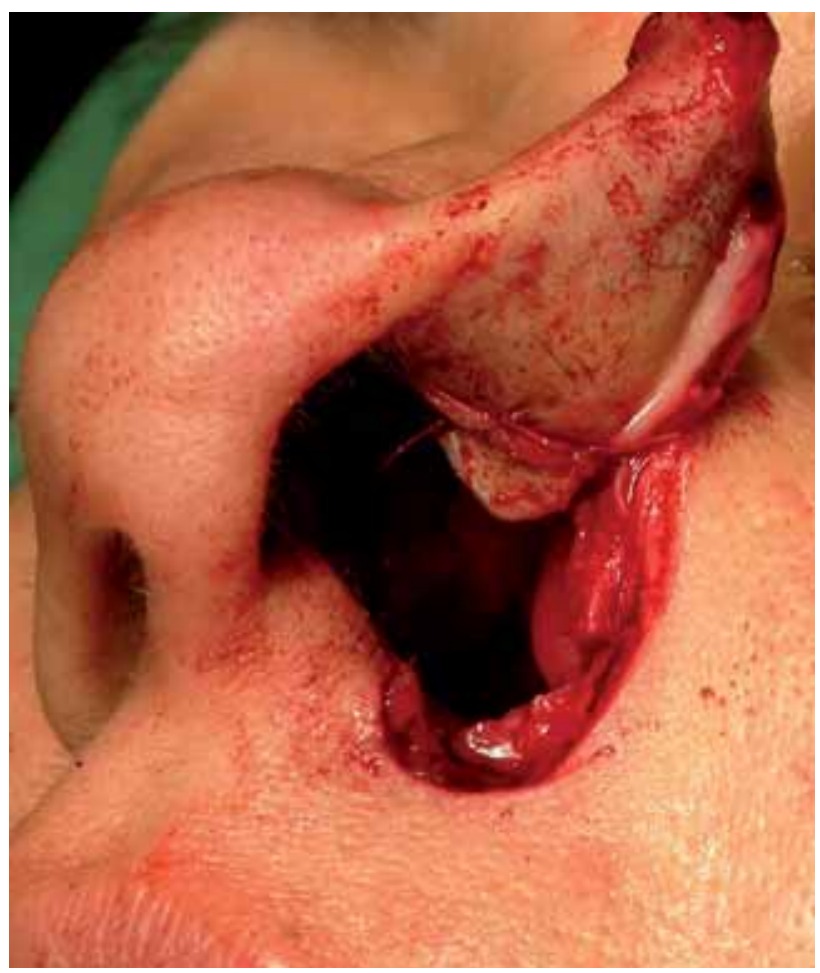

Figure 2. Intra-operative images showing the lateral rhinotomy.

Anterior rhinoscopy revealed an ulcerated mass located at the left nasal vestibule (Figure 1). The rest of the otolaryngological examination was unremarkable. Subsequently the patient was investigated with a head and neck computed tomography scan. There was no evidence of erosion of the adjacent bone or any cervical lymph node enlargement. All evidence suggested that the disease was localized at the left nasal vestibule. In view of the results the patient was staged as T2 N0 M0 according to the American Joint Committee on Cancer (AJCC) TNM classification of Malignant Tumours ${ }^{(3)}$. The management plan was decided following discussion in the multidisciplinary Head and Neck Oncology meeting (HNMDM).

The patient underwent excision of the lesion with a left lateral rhinotomy approach and reconstruction of the surgical defect with a left conchal graft (Figures 2 and 3). In the absence of clinical or radiological evidence of cervical lymph node involvement, no prophylactic treatment was administered to the neck.

Histopathological examination revealed Acinic Cell carcinoma arising in a minor salivary gland, which was excised to clear margins (Figure 4). The patient has been followed-up for almost three years, and no lateral nasal wall collapse, local recurrence or regional metastasis has been encountered thus far.

\section{DISCUSSION}

Malignant tumours of the nose and paranasal sinuses are considered rare neoplasms, which represent approximately $3 \%$ of

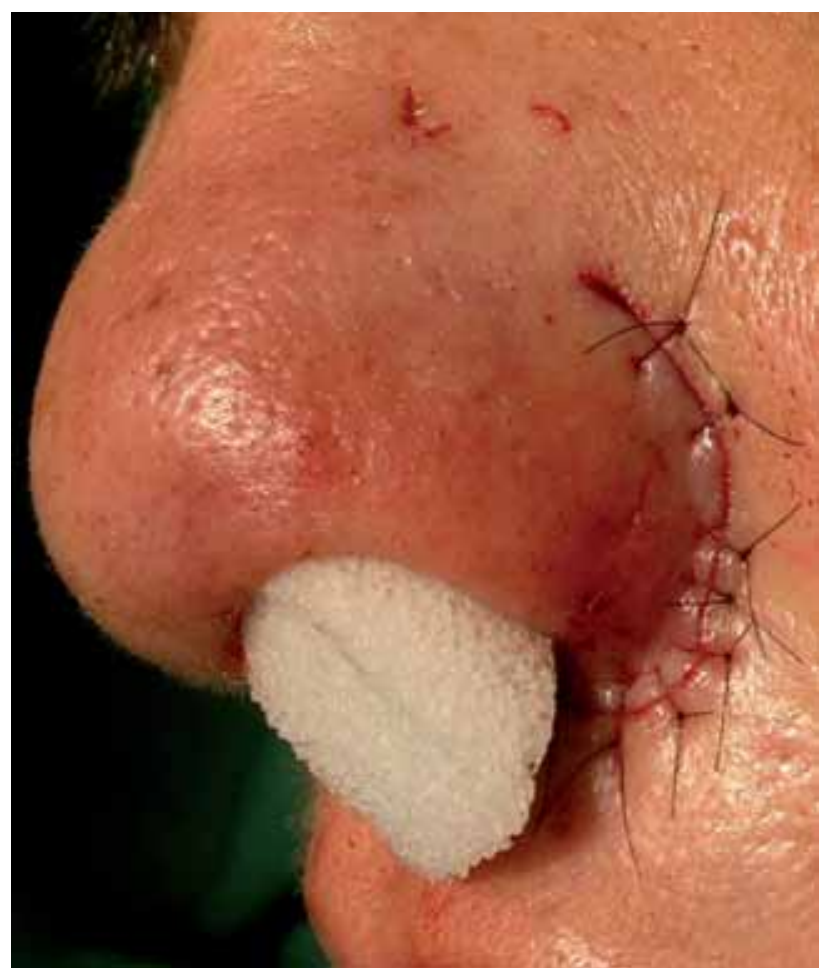

Figure 3. Intra-operative images showing the conchal graft used for reconstruction of the nose.

all head and neck malignancies and only a fraction of these arise from the nasal cavity ${ }^{(4)}$. Sinonasal malignancies are seen predominantly in the fifth to sixth decades of life. The incidence in males is twice that in females. Published data demonstrated that approximately $50 \%$ of all cancers of the nasal cavity are squamous cell carcinomas (SCC) ${ }^{(5)}$.

Our literature research revealed only 15 documented cases of sinonasal ACC (Summarized in Table 1) ${ }^{(6-17) .}$ ACC is a lowgrade neoplasm that mainly affects the parotid gland, but

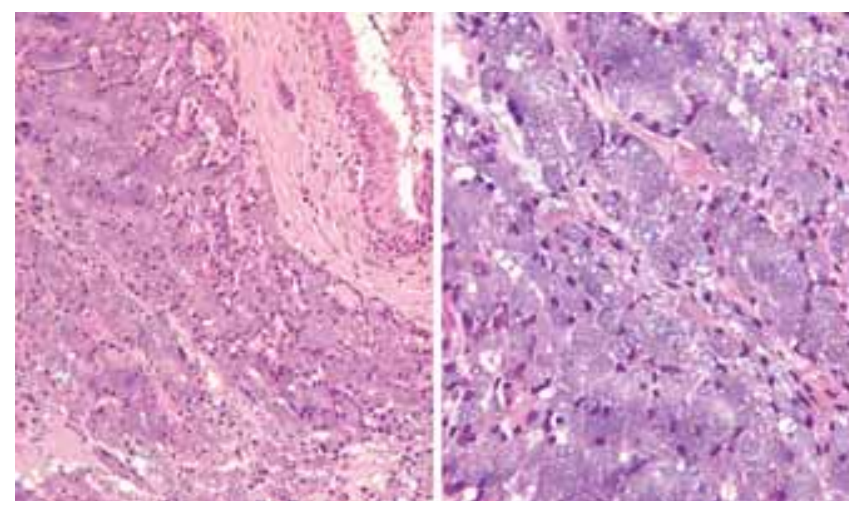

Figure 4. Photomicrographs of histology slides confirming acinic cell carcinoma of salivary gland type, showing a solid growth pattern. Left: Low power view showing sheets of well organised acini covered by respiratory epithelium (H\&E, 200X). Right: High power view showing well differentiated acinar cells, with infrequent mitoses. The cytoplasm is granular and lightly basophilic; nuclei are eccentrically positioned. (H\&E, 400X). 
Table 1. Sixteen documented cases of sinonasal acinic cell carcinoma.

Abbreviations: M, male; F, female; Rt, right; Lt, left; S, surgery; RT, radiotherapy; N/S, not specified; NED, no evidence of disease; Y, years

\begin{tabular}{|c|c|c|c|c|c|c|}
\hline Source & $\begin{array}{l}\text { Patient } \\
\text { Sex/Age }\end{array}$ & Tumour site & $\begin{array}{c}\text { Size } \\
\text { (in cm) }\end{array}$ & $\begin{array}{l}\text { Presenting } \\
\text { Symptoms }\end{array}$ & Treatment & Follow-up \\
\hline Manace et al, $1971^{(6)}$ & F/49 Y & $\begin{array}{l}\text { Lt ethmoid + } \\
\text { maxillary sinus }\end{array}$ & $\mathrm{N} / \mathrm{S}$ & Obstruction & S & NED $1.3 \mathrm{Y}$ \\
\hline Spiro et al, $1978^{(7)}$ & N/S Y & $\mathrm{N} / \mathrm{S}$ & $\mathrm{N} / \mathrm{S}$ & $\mathrm{N} / \mathrm{S}$ & $\mathrm{N} / \mathrm{S}$ & $\mathrm{N} / \mathrm{S}$ \\
\hline Perzin et al, $1981^{(8)}$ & $\mathrm{F} / 75 \mathrm{Y}$ & Lt inferior turbinate & 3.0 & Obstruction, Epistaxis & $\mathrm{S}$ & $\mathrm{N} / \mathrm{S}$ \\
\hline Ordonez et al, $1986^{(9)}$ & $\mathrm{F} / 60 \mathrm{Y}$ & Rt superior meatus & 2.0 & Nasal polyp, Epistaxis & $\mathrm{S}$ & NED $7 \mathrm{Y}$ \\
\hline Finkelhor et al, $1987^{(10)}$ & F/45 Y & Septum & $\mathrm{N} / \mathrm{S}$ & Obstruction & S & $\mathrm{N} / \mathrm{S}$ \\
\hline Hanada et al, $1988^{(11)}$ & $\mathrm{M} / 68 \mathrm{Y}$ & Rt inferior turbinate & 6.0 & Obstruction & $\mathrm{S}+\mathrm{RT}$ & NED $3 \mathrm{Y}$ \\
\hline Takimoto et al, $1989^{(12)}$ & $\mathrm{F} / 60 \mathrm{Y}$ & $\begin{array}{l}\text { Middle and } \\
\text { inferior turb }\end{array}$ & $\mathrm{N} / \mathrm{S}$ & Nasal polyp, Epistaxis & S & NED 2 Y \\
\hline Valerdiz-Casasola et al, $1993^{(13)}$ & $\mathrm{M} / 47 \mathrm{Y}$ & Lt nasal Cavity & 2.5 & Obstruction, Epistaxis & $\mathrm{S}+\mathrm{RT}$ & NED 10/12 \\
\hline Schmitt et al, $1994^{(14)}$ & $\mathrm{M} / 60 \mathrm{Y}$ & Rt inferior turbinate & $\mathrm{N} / \mathrm{S}$ & Obstruction & $\mathrm{N} / \mathrm{S}$ & $\mathrm{N} / \mathrm{S}$ \\
\hline von Biberstein et al, $1999^{(15)}$ & $\mathrm{F} / 76 \mathrm{Y}$ & Rt middle turbinate & $\mathrm{N} / \mathrm{S}$ & Nasal polyp & $\mathrm{S}$ & NED $3 \mathrm{Y}$ \\
\hline Sapci et al, $2000^{(16)}$ & M/47 Y & Lt septum & 1.0 & Nasal polyp, Epistaxis & $\mathrm{S}$ & NED $1.8 \mathrm{Y}$ \\
\hline Neto et al, $2005^{(17)}$ & $\mathrm{M} / 50 \mathrm{Y}$ & Nasal Cavity & $\mathrm{N} / \mathrm{S}$ & Obstruction (polyp) & $\mathrm{S}+\mathrm{RT}$ & $\begin{array}{l}1 \text { Recurrence, } \\
\text { NED } 12 \mathrm{Y}\end{array}$ \\
\hline Neto et al, $2005^{(17)}$ & $\mathrm{F} / 42 \mathrm{Y}$ & Lt inferior turbinate & 2.0 & Obstruction (polyp) & $\mathrm{S}$ & NED $7 \mathrm{Y}$ \\
\hline Neto et al, $2005^{(17)}$ & $\mathrm{M} / 65 \mathrm{Y}$ & Rt inferior turbinate & 2.5 & Obstruction & $\mathrm{S}$ & NED $4 \mathrm{y}$ \\
\hline Manganaris et al, 2008 & $\mathrm{~F} / 51 \mathrm{Y}$ & Lt nasal Vestibule & 1.5 & Localized Pain & $\mathrm{S}$ & NED $2.7 \mathrm{Y}$ \\
\hline
\end{tabular}

occurs only rarely in minor salivary glands. ACC comprises only $0.3 \%$ to $0.5 \%$ of all minor salivary neoplasms ${ }^{(1,15)}$. This neoplasm will typically present in the 5th decade of life and shows a predilection for women and Caucasians ${ }^{(7,18,19) .}$ In the sinonasal tract, the ages in reported cases range from 42 to 76 years (median, 57 years) and there appears to be no sex predilection (Table 1).

Salivary ACC consists of lobules of round uniform-appearing cells with abundant cytoplasm arranged in nests ${ }^{(18,19)}$. Tumour cells commonly resemble the serous acinar cells of the parotid gland, are dark staining and have granular or honeycomb cytoplasm ${ }^{(18,19)}$. Microscopic appearance has been categorized as solid, microcystic, papillary cystic, lobular and follicular ${ }^{(18,19)}$. The predominant variant is the solid growth with similar histopathological features to those of the present case.

Early symptoms in most sinonasal tumours differ very little from benign sinonasal disease. Common presenting symptoms include nasal obstruction, epistaxis, nasal discharge or recurrent infection, headache, facial swelling or localised pain, unilateral diplopia, epiphora or proptosis, and cranial neuropathies. A probable temporary regression of these symptoms after administration of antibiotics or local decongestants inevitably results in a false reassurance of both patients and doctors and a delayed diagnosis of a malignancy. In the reported cases of sinonasal ACC the commonest presenting complaint was unilateral nasal obstruction (71\%), followed by epistaxis (36\%).

Meticulous pre-treatment evaluation and staging of sinonasal tumours, as well as multidisciplinary pre-treatment assessment are of paramount importance and are consistently practised in our unit. Staging of nasal cavity malignancies was not as well established as for other head and neck tumours. Several studies have underlined their reservations with regards to the suitability of the TNM classification system for tumours of the nasal vestibule. In contrast to the nasal cavity, which is lined with pseudostratified columnar ciliated epithelium, the nasal vestibule is lined with squamous epithelium and contains vibrissae as well as sweat and sebaceous glands. Patients with nasal vestibule primary tumours are often excluded from large reviews of sinonasal malignancies, on the grounds that these tumours are probably related more to skin primary tumours than to true primary nasal malignancies ${ }^{(4)}$.

An independent classification system for this sub site of the nasal cavity has been devised by Wang, which he advocated as being more accurate compared to the TNM staging system ${ }^{(20)}$. According to this system, T1 is used when the tumour is confined to skin, T2 when there is invasion of the subcutaneous tissue and cartilage and T3 when there is bone involvement. Recently, cancer of the nasal cavity has been added to the AJCC staging system ${ }^{(3)}$. Under the section for tumours of the paranasal sinuses, nasal cavity and ethmoid sinus tumors are classified separately. The nasal cavity is further divided into 4 sub-sites: septum, floor, lateral walls, and vestibule.

Principles of management for malignancies of the nasal vestibule vary depending on tumour staging, and commonly include surgery and radiotherapy. The major disadvantage of surgery is a potentially sub-optimal functional or cosmetic result depending on the extent of surgery. In nasal reconstruction, the auricular concha provides a graft, which is easy to shape and generally offers the possibility to reconstruct various 
anatomical components of the nasal pyramid with satisfactory functional and cosmetic results. The use of autologous cartilage still provides the best resistance to infection and a low degree of resorption. For all the above reasons the conchal graft was also favored in the nasal reconstruction of the present case.

Radiotherapy remains an important adjuvant in the treatment of malignancies of the nasal vestibule and has occasionally been used effectively as the single modality of treatment. However, this is not necessarily recommended since radiation of the nose has significant morbidity. In the reported cases of sinonasal ACC, adjuvant RT was used in 3 cases, one of which was for local failure. Local failure is generally reported as the most frequent type of recurrence for malignancies of the nasal vestibule.

Treatment of acinic cell carcinoma of salivary gland type includes surgical excision with a margin of uninvolved tissue ${ }^{(7,18,19)}$. Post-operative radiation therapy is reserved for cases of questionable residual disease after surgery or local recurrence. Early survival rates are quoted as high as $76 \%$ at 5 years, $63 \%$ at 10 years but only at $55 \%$ at 15 years after treatment ${ }^{(7)}$. This is due to the tumour's tendency for local recurrence or distant metastasis many years after initial treatment ${ }^{(7,18,19)}$. For patients with sinonasal ACC, prognosis appears to be associated with the extent of disease at the time of initial treatment and the adequacy of the resection ${ }^{(6-17)}$.

\section{CONCLUSION}

The histology is varied in the nasal cavity, which explains the diverse pathology that presents itself here. This paper discussed the considerations in the evaluation and management of an exceptionally rare malignancy that arose in the nasal cavity, acknowledging the fact that specific management decisions for rare clinical entities cannot be made from a single case report.

The treatment regimen for sinonasal acinic cell carcinomas remains empirical and possibly controversial due to insufficient clinical data. It is fair to say that each reported case was addressed on an individual basis. The best result is often achieved when each patient is individually managed based on expected local control rates, functional and cosmetic outcome, risk of complications, and individual experience.

\section{REFERENCES}

1. Spiro RH. Salivary neoplasms: overview of a 35 year experience with 2,807 patients. Head Neck Surg 1986; 8: 177-184.

2. Goepfert H, Luna MA, Lindberg RD, White AK. Malignant salivary gland tumors of the paranasal sinuses and nasal cavity. Arch Otolaryngol 1983; 109: 662-668.

3. American Joint Committee on Cancer. AJCC Cancer Staging Manual. 6th ed. New York, NY: Springer-Verlag; 2003.
4. Dulguerov P, Jacobsen MS, Allal AS, Lehmann W, Calcaterra T. Nasal and Paranasal Sinus Carcinoma: Are We Making Progress? A Series of 220 Patients and a Systematic Review. Cancer. 2001; 92: 3012-3029.

5. Bhattacharyya $\mathrm{N}$. Cancer of the nasal cavity: survival and factors influencing prognosis. Arch Otolaryngol Head Neck Surg 2002; 128: 1079-1083.

6. Manace ED, Goldman JL. Acinic cell carcinoma of the paranasal sinuses. Laryngoscope 1971; 81: 1074-1082.

7. Spiro RH, Huvos AG, Strong EW. Acinic cell carcinoma of salivary origin. A clinicopathologic study of 67 cases. Cancer 1978; 41: 924-935.

8. Perzin KH, Cantor JO, Johannessen JV. Acinic cell carcinoma arising in nasal cavity. Report of a case with ultrastructural observations. Cancer 1981; 47: 1818-1822.

9. Ordonez NG, Bataskis JG. Acinic cell carcinoma of the nasal cavity: electron optic and immunohistochemical observations. J Laryngol Otol 1986; 100: 345-349.

10. Finkelhor BK, Maves MD. Pathology quiz case 1. Arch Otolaryngol Head Neck 1987; 113: 1120-1122.

11. Hanada T, Moriyama I, Fukami K. Acinic cell carcinoma originating in the nasal cavity. Arch Otorhinolaryngol 1988; 245: 344-347.

12. Takimoto T, Kano M, Uneda R. Acinic cell carcinoma of the nasal cavity. A case report. Rhinology 1989; 27: 191-196.13.

Valerdiz-Casasola S, Sola J, Pardo-Mindan FJ. Acinic cell carcinoma of the nasal cavity with intracytoplasmic crystalloids. Histopathology 1993; 23: 382-384.

14. Schmitt FC, Wal R, Cunha-Santos G. Acinic cell carcinoma arising in nasal cavity. Diagnosis by fine-needle aspiration. Diagn Cytopathol 1994; 10: 96-97.

15. von Biberstein SE, Spiro JD, Mancoll W. Acinic cell carcinoma of the nasal cavity. Otolaryngol Head Neck Surg 1999; 120: 759-762.

16. Sapci T, Yildirim G, Peker K, et al. Acinic cell carcinoma originating in the nasal septum. Rhinology 2000; 38: 140-143.

17. Neto AG, Pineda-Daboin K, Spencer ML, Luna MA. Sinonasal acinic cell carcinoma: a clinicopathologic study of four cases. Head Neck. 2005; 27: 603-607.

18. Barnes L, Eveson JW, Reichert P, Sidransky D, eds: World Health Organization Classification of Tumours. Pathology and genetics of head and neck tumours. Lyon: IARC Press 2005: 216-218.

19. Ellis GL, Auclair PL. Tumors of salivary glands. In: Atlas of Tumor Pathology. Vol 3. Washington, DC: Armed Forces Institute of Pathology; 1995; pt 17: 183-203.

20. Wang CC. Treatment of carcinoma of the nasal vestibule by irradiation. Cancer. 1976; 38:100-106.

Argyris Manganaris PhD, DOHNS (RCSEng) Department of Otorhinolaryngology Head and Neck Surgery

3rd Floor Southwark Wing, Guy's Hospital

Great Maze Pond,

London, SE1 9RT

United Kingdom

Tel: +44-207-188-2216,

Fax: +44-207-188-2206

E-mail: amanganaris@hotmail.com 